\title{
Preliminary Study of an Integral Harmonic Analysis Magnetic Field Measurement System for Long SSC Magnets*
}

\author{
Michael I. Green \\ Lawrence Berkeley Laboratory \\ University of California, 7/222 \\ Berkeley, California 94720
}

\section{Abstract}

We describe the research and development required to design and build a prototype system capable of making integrated magnetic multipole measurements of warm and cryogenic 50 $\mathrm{mm}$ bore SSC dipole and quadrupole magnets utilizing a warm probe in a warm finger. Our experience and some preliminary studies indicate that it is highly unlikely that a 16 meter long probe can be fabricated that will have a twist below several milliradians at any temperature. Consequently we describe a segmented 16 meter long probe, for which we intend to calibrate the phase of each segment to within 0.1 milliradians. The data for all segments will be acquired simultaneously, and integrated data will be gencrated from the vector sums of the individual segments. The calibration techniques and instrumentation required to implement this system are described. The duration of an integral measurement at one current is less than ten seconds, which is three orders of magnitude shorter than that required by the mole technique presently being used. The system is based on an extrapolation of the techniques used at LBL to measure cryogenic 1 meter models of SSC magnets with a cryogenic probe.

\section{INTRODUCTION}

This work is based upon an earlier proposal to the Central Design Group of the SSC [1]. The earlier proposal was for a cryogenic probe. Opening up the bore diameter of SSC dipoles from 40 to $50 \mathrm{~mm}$ makes a "warm-finger" device (i.e., an insulated room temperature probe) practical and cost effective.

The short duration of the data acquisition cycle has at least two benefits, it will shorten the time needed for measurements and it should allow measurements of the decay of persistent currents during an injection cycle.

\section{ECONOMICS}

A strong advantage of this system over the "mole" system presently being used is that the time required for an integral measurement is less than 10 seconds, whereas the mole now

\footnotetext{
*This work was supported by the Director, Office of Energy Research, Office of High Energy and Nuclear Physics, Division of High Energy Physics, of the U.S. Department of Energy under Contract No. DE-AC03-76SF00098.
}

requires a few hours to make an integral measurement. This can lead to significant savings.

\section{A. Room Temperature Testing}

If 8000 dipoles are measured at room temperature at currents of plus and minus ten Amperes, and the mole requires two hours for a measurement at one current, $\$ 200$ per hour testing costs results in a saving of $\$ 6,400,000$.

\section{B. Cryogenic Testing}

If 800 dipoles are measured, at two hours per current with six currents, $\$ 300$ per hour testing results in a saving of $\$ 2,880,000$.

\section{Research and Development Tests}

The above savings do not include the large number of measurements that will be required to characterize the dipoles and also ensure that there is correspondence between cryogenic and room temperature measurements.

It is highly probable that faster measurements will decrease the number of test stands required resulting in additional savings.

\section{MEASUREMENT PROBE}

Figure 1 illustrates the measurement probe with its associated drive, encoders and gravity sensors. The search coil array will be 16 meters long and will consist of five to sixteen identical segments depending upon SSC specifications for twist and magnetic field axial structure.

\section{A. Probe Cross-section}

Figure 2 portrays cross-sections of both radial and tangential dipole search coil arrays. Each segment will have coils to mcasure the dipole strength and the higher error harmonics. The error harmonics will be measured utilizing coils connected in series opposition implementing analog bucking of the dipole signal. Additional coils have been included for mechanical symmetry in order to reduce stresses that will produce bowing of the search coil array. The gaps between the ends of segments will be small in comparison to the segment length and the integrated data will be corrected for the gaps. 


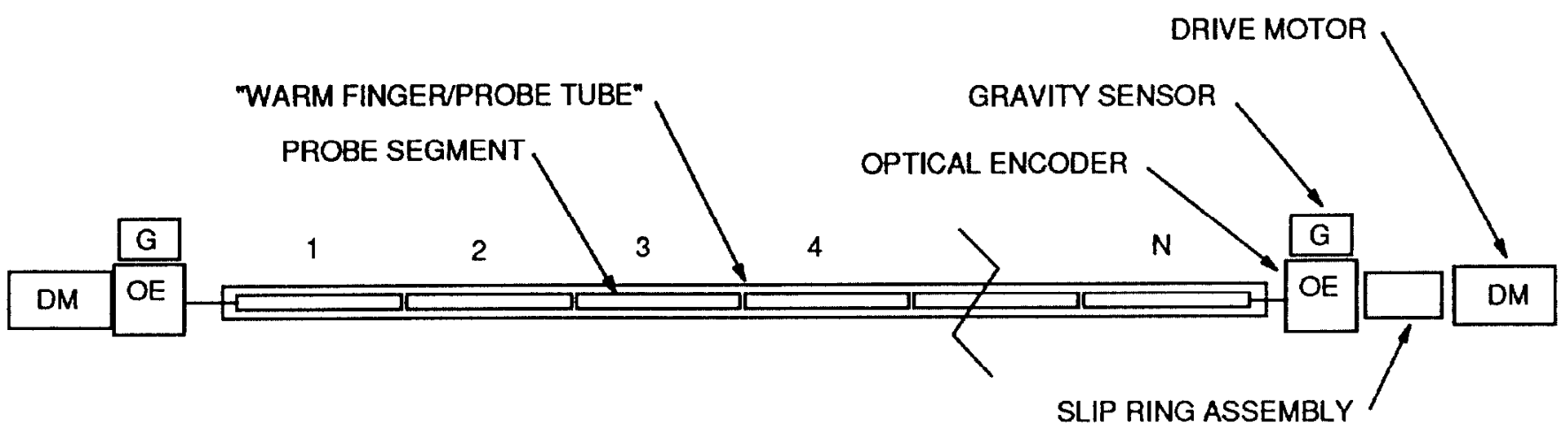

Figure 1. Measurement Probe System.

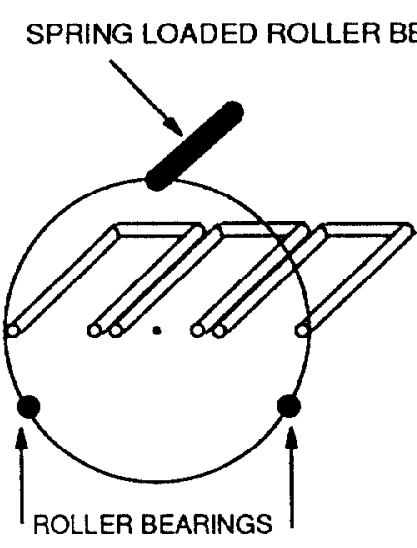

RADIAL COIL ARRAY

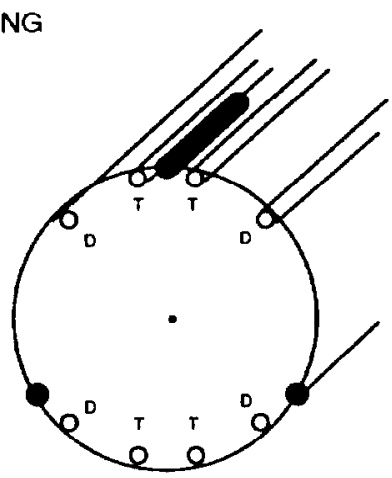

TANGENTIAL COIL ARRAY
Figure 2. Cross-section of search coil array options. Each array has the capability for bucking the dipole signal during harmonic measurements. For symmetry each array has dummy coils.

Tangential coils/coil support will weigh less and will allow a higher degree of probe symmetry than radial coils. Radial coils can be made structurally more symmetrical at the expense of added dead weight (i.e. increased probe twist). Radial coils can be fabricated with a much higher sensitivity than tangential coils and since each segment would have three identical coils, it might be cost effective to mass produce them using printed circuit board techniques.

The pros and cons of tangential versus radial coils will be investigated.

\section{B. Probe Support}

The mechanically simplest scheme consists of three circumferentially installed shaft mounted rollers per support. Two of the rollers are fixed, and the third is radially spring loaded. The fixcd rollers serve to center the probe in the magnet bore tube, while the spring-loaded roller provides the necessary force to accomplish this task. This scheme is inherently free of radial backlash.

\section{Twist Calibration}

Each search coil array will be in its "warm-finger "or warm magnet "bore tube." A dipole reference magnet (with length slightly longer than a segment) will be positioned at each coil segment along the bore tube, always keeping a constant orientation of the dipole magnet with respect to gravity. Data will be acquired: 1) with both clockwise and counterclockwise rotations of the probe, 2 ) with the probe driven from each end separately, and 3) with the dipole reference magnet rotated about its vertical axis. These eight sets of data for each position will allow the calibration of both the twist and the absolute phase of each segment of the search coil array with respect to gravity. For this proposal to be most effective, it will be necessary that the twist be reproducible. Absolute optical encoders at each end of the probe will allow monitoring of twist reproducibility.

After the twist has been calibrated, the option of making measurements continuously in one direction is viable. This would enable very rapid measurements during current ramping. It would also allow good measurements of the decay of persistent currents during an injection cycle.

\section{DATA ACQUISITION SYSTEM}

\section{A. Computer Hardware}

The computer system will consist of a UNIX workstation connected by means of ETHERNET to a VXI crate. The VXI crate incorporates interfaces to CAMAC and IEEE-488 bus's if the applicable VXI modules can not be acquired.

\section{B. Instrumentation}

Figure 3 is a representative block diagram of the instrumentation required to acquire the harmonic analysis data. Other instrumentation, such as a DVM, multiplexer, temperature monitors, power supply controller, gravity sensor monitors, etc., will also be required. 


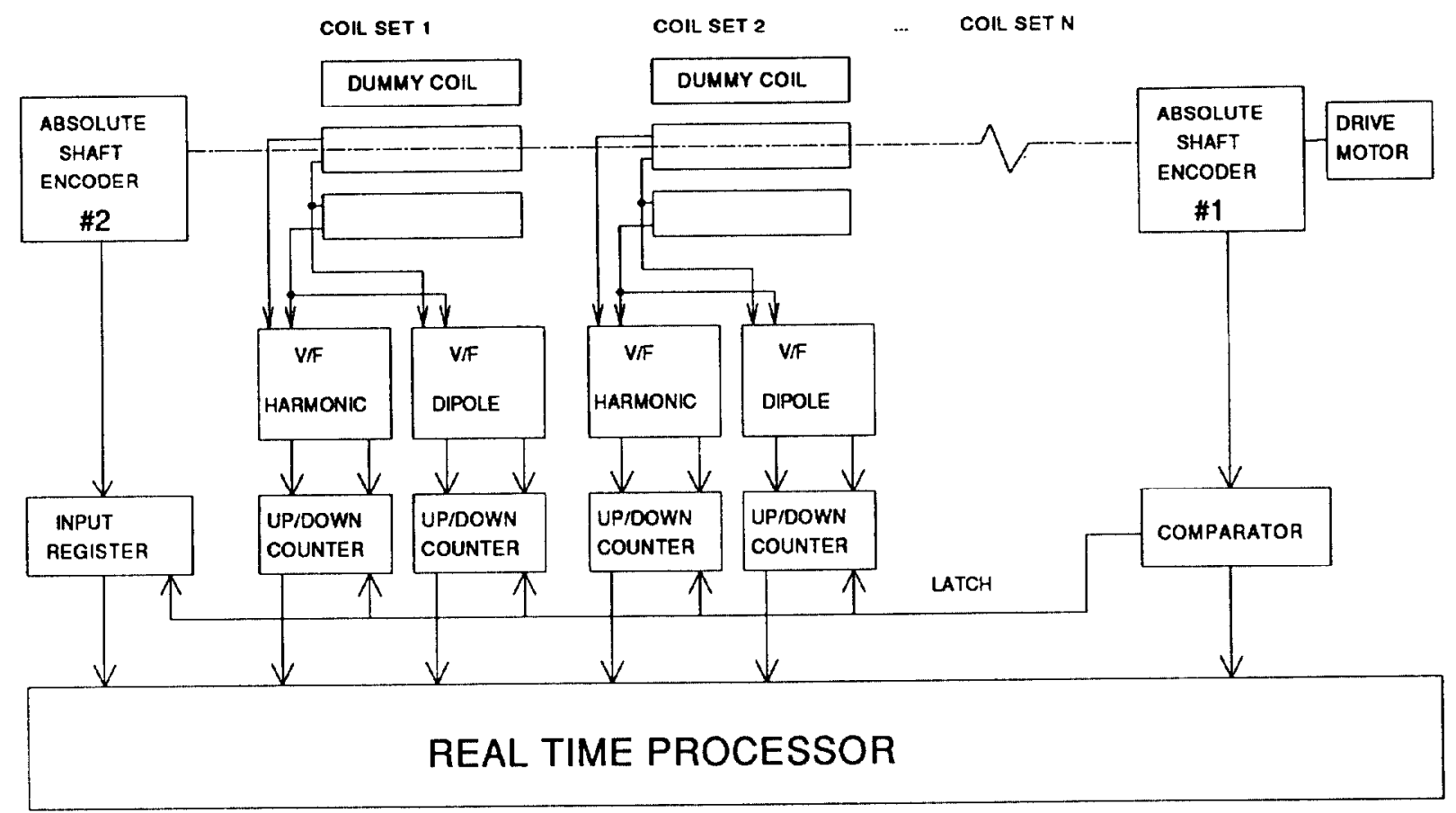

Figure 3. Harmonic analysis electronic instrumentation.

Each probe segment will have two digital integrators enabling simultaneous acquisition of unbucked and bucked data. Figure 3. Harmonic analysis electronic instrumentation.

The digital integrators will be hardware-latched by an optical encoder at the motor end of the search coil array. An optical cncoder at the other end of the array will provide information as to the total twist of the array. There will also be a gravity reference at each end.

\section{Computer Software}

$V x W o r k s$ is a real-time operating system that is downloaded to the VXI crate from the UNIX Workstation [2]. UNIX is used as a high-level software development platform to develop real-time code that will run and be debugged under $\mathrm{VxW}$ Wrks. The application code will be structured similar to the system developed at LBL for the Supercon group [3].

\section{Data Format}

The output data format will be compatible with the SelfDescribing Data set software developed by the SSC CDG.

\section{COST ESTIMATE AND SCHEDULE}

\section{A. Prototype}

It is not anticipated that this system would require significant research. Most of the development would be application of known technology.
A system with the same precision as the BNL "mole" could be developed at a cost of $\$ 500,000$ within 12 months.

\section{B. Additional Probes}

Additional probes including drive, gravity sensors and encoders are estimated to cost under $\$ 100,000$.

Additional data acquisition systems are estimated to also cost under $\$ 100,000$. This system would include the measurement probe system, data acquisition system and software.

\section{ACKNOWLEDGEMENTS}

Leif Hansen did the mechanical engineering work for the earlier cryogenic probe proposal.

\section{REFERENCES}

[1] M. 1. Green, L. Hansen, "Proposal for a cryogenic magnetic field measurement System for SSC dipole magnets," to be published in the Proceedings of the Third Annual International Industrial Symposium on the Super Collider, Atlanta, GA, March 13-15, 1991.

[2] Wind River Systems, 1010 Atlantic Avenue, Alameda, CA 94501

[3] M. I. Green, P. J. Barale, W. S. Gilbert, W. V. Hassenzahl, D. H. Nelson, C. E. Taylor, N. J. Travis, and D. A. Van Dyke, "Magnetic Measurements System for Harmonic Analysis of LBL SSC Model Dipoles and Quadrupoles," presented at the Tenth International Conference on Magnet Technology, September 21-25, 1987, Boston, Massachusetts, IEEE Transactions on Magnetics, Vol. 24, No. 2, p.958 (March 1988). 